\title{
Características químico-sensoriales de los aceites de oliva "Arbequina» obtenidos en distintas zonas de España
}

\author{
Por J. Tous', A. Romero', J. Plana1, L. Guerrero², I. Díaz² y J. F. Hermoso1 \\ Institut de Recerca i Tecnologia Agroalimentaria (IRTA) \\ 1 Centre de «Mas Bové». Apartat 415. 43280 Reus (Tarragona) \\ 2 Divisió Alimentària-CTC. Granja Camps i Armet. 17121 Monells (Girona)
}

\section{RESUMEN}

Características químico-sensoriales de los aceites de oliva "Arbequina» obtenidos en distintas zonas de España

Dentro de los aceites de oliva vírgenes de calidad que se producen en España, Cataluña destaca por tener dos denominaciones de origen (Garrigues y Siurana) de las seis actualmente existentes. La producción de aceite virgen extra de las mismas procede de la variedad "Arbequina", aproximadamente $8.000 \mathrm{tm}$, repartidas entre las D. O. "Garrigues», en Lleida $(5.000 \mathrm{tm})$, y "Siurana" en Tarragona (3.000 tm). Estos aceites son muy apreciados tanto a nivel nacional como internacional por sus excelentes características organolépticas. En los últimos años, esta variedad se está extendiendo por España (Andalucía, Aragón, etc.) y otros países del mundo (Argentina, Francia, etc.).

Se estudian las características físico-químicas y sensoriales de los aceites de oliva vírgenes de la variedad "Arbequina" producidos, principalmente, en Cataluña, Andalucía y Aragón. Los resultados preliminares obtenidos indican que algunos parámetros de aceite de "Arbequina" varían significativamente al cultivarla en diferentes medios agrológicos, principalmente, determinados ácidos grasos, polifenoles, estabilidad y algunos atributos sensoriales del aceite.

PALABRAS-CLAVE: Aceite de oliva virgen - Calidad Características físico-químicas - Características sensoriales Influencia del medio - Variedad arbequina.

\section{SUMMARY}

Chemical and sensory characteristics of "Arbequina» olive oil obtained in different growing areas of Spain

Within the quality virgin olive oils produced in Spain, Catalonia has two of the six currently existing Origin Denominations (Garrigues and Siurana). Their production of extra virgin oil proceeds of the "Arbequina" cultivar, approximately $8.000 \mathrm{tm}$, distributed between the OD "Garrigues", in Lleida $(5.000 \mathrm{tm})$, and "Siurana" in Tarragona $(3.000 \mathrm{tm})$. These oils are very appreciated at domestic as well as at international level because of their excellent taste. In the last years, this variety is being expanded in other regions of Spain (Andalusia, Aragon, etc.) and over the world (Argentina, France, etc.).

The physico-chemical and sensory characteristics of "Arbequina" virgin olive oils produced are studied, mainly, in Catalonia, Andalusia and Aragon. The obtained preliminary results indicate that some quality parameters of the "Arbequina" oil are variable, being cultivated in different environments, mainly certain fatty acids, polyphenols, oxidative stability and some sensory attributes of the oil.

KEY-WORDS: Arbequina variety - Environmental effects Physico-chemical characteristics - Quality - Sensory characteristics - Virgin olive oil.

\section{INTRODUCCIÓN}

Dentro de los aceites de oliva vírgenes de calidad que se producen en España, incluidos en las seis denominaciones de origen actualmente existentes, Cataluña destaca por tener dos de ellas, Garrigues y Siurana. La producción de aceite virgen extra de las mismas procede de la variedad "Arbequina», aproximadamente $8.000 \mathrm{tm}$, repartidas entre las D. O. «Garrigues», en Lleida (5.000 tm) y «Siurana» en Tarragona (3.000 tm). Estos aceites son muy apreciados en el mercado, tanto a nivel nacional como internacional, por sus excelentes características organolépticas $y$, tradicionalmente, se exportan unas 3.000 tm a la UE (Italia) para encabezar otros aceites de oliva. En los últimos años, esta variedad se está extendiendo por otras regiones de España (Andalucía, Aragón, etc.) y otros países del mundo (Argentina, Francia, etc.).

Los aceites vírgenes de «Arbequina» producidos en Cataluña se han caracterizado, tradicionalmente, por tener unos excelentes atributos sensoriales y unas características físico-químicas propias. Estas últimas, principalmente la composición en ácidos grasos, polifenoles y estabilidad, se ha comprobado en distintos trabajos realizados en el IRTA-Mas Bové, que varían en función de la zona de cultivo, principalmente debido a las condiciones edafoclimáticas (Tous y Romero, 1993 y 1994 a; Romero et al., 1995), también tiene influencia el nivel de cosecha del árbol y el grado de maduración de la aceituna. 
La mayoría de variedades de olivo presentan, en general, una composición en ácidos grasos característica, con independencia del medio en que están cultivadas (Cucurachi, 1965; Uceda y Frías, 1975; Humanes y Civantos, 1993; Uceda y Hermoso, 1997), tal es el caso de "Picual», "Hojiblanca», «Frantoio» y "Leccino". Sin embargo, existen también trabajos, parecidos a los del IRTA, que analizan la interacción cultivar/ambiente (Cimato, 1990; Parlati et al., 1992; Angerosa et al., 1996) y el efecto de la altitud en la composición de los aceites vírgenes (Ferreiro y Aparicio, 1992; Moussa et al., 1996), poniendo de manifiesto, en algunas variedades, que tiene mayor influencia el factor agrológico (localidad, clima, técnicas de cultivo, etc.) que el genético en las variaciones de algunos componentes del aceite, destacando determinados ácidos grasos, esteroles, escualeno, triglicéridos, fitol, etc. Otros estudios relacionados con lo expuesto, englobados dentro del proyecto SEXIA (Sistema Experto para la Identificación de Aceites) que desarrolla el Instituto de la Grasa de Sevilla, pretenden identificar un aceite por sus características químicas y sensoriales, para poder identificar la variedad y su origen geográfico, asociándole para ello a cada hipótesis un valor de posibilidad o probabilidad (Aparicio et al., 1988; Aparicio et al., 1991; Aparicio, 1995).

La estabilidad oxidativa es otro parámetro definitorio de la calidad del aceite virgen, estando muy influenciado por la variedad (Uceda et al., 1989; Tous y Romero, 1993) y el grado de maduración de la aceituna (García et al., 1996). En el contenido de polifenoles la influencia varietal es menor, mostrándose muy variable en las distintas campañas, nivel de cosecha, grado de maduración de la aceituna, altitud, efecto del riego, etc. (Uceda et al., 1989; Cimato et al., 1990; Inglese, 1994; Beltrán et al., 1995; Moussa et al., 1996). En cuanto a las características sensoriales del aceite, se ha observado que algunos atributos (amargor, dulzor, etc.) pueden variar en función de la cosecha (Solé, 1995), maduración de la aceituna (García et al., 1996) y zona de cultivo (Angerosa et al., 1995; Uceda y Hermoso, 1997).

En el presente trabajo, teniendo en cuenta los antecedentes bibliográficos, se pretende profundizar en el estudio de la variabilidad de algunas características físico-químicas y sensoriales de los aceites vírgenes de "Arbequina» obtenidos en distintos medios agrológicos de Cataluña, Andalucía y Aragón.

\section{PARTE EXPERIMENTAL}

Durante los años 1994 a 1996 y en distintas fases de maduración (15 y 30 de noviembre; 15 y 30 de diciembre), se recogieron un total de 107 muestras de aceite de "Arbequina» en almazaras de distintas zonas de España: D. O. Garrigues y Siurana, otras

Tabla I

Caracterización de las muestras del aceite virgen de «Arbequina» según su procedencia, cosecha y épocas de recogida, y de los análisis realizados

\begin{tabular}{|c|c|c|c|c|c|c|c|}
\hline \multirow{2}{*}{$\begin{array}{l}\text { Comunidad } \\
\text { Cataluña }\end{array}$} & \multirow{2}{*}{$\begin{array}{c}\begin{array}{c}\text { Zona } \\
\text { (provincia) }\end{array} \\
\text { Siurana-Camp (T) }\end{array}$} & \multirow{2}{*}{$\begin{array}{l}\text { Localidad } \\
\text { Cambrils, Montbrió, Montroig } \\
\text { Riudecanyes, Riudoms, La Selva, Valls }\end{array}$} & $\begin{array}{l}\text { Épocas de } \\
\text { recogida }\end{array}$ & $\begin{array}{c}\text { Años } \\
1994\end{array}$ & \multirow{2}{*}{$\begin{array}{c}\begin{array}{c}\text { Muestras } \\
(n .9)\end{array} \\
23\end{array}$} & \multicolumn{2}{|c|}{$\begin{array}{l}\text { Análisis } \\
\text { Químicos Sensoriales }\end{array}$} \\
\hline & & & 4 & $\begin{array}{l}1994 \\
1995 \\
1996\end{array}$ & & 23 & 16 \\
\hline & Siurana-Montsant $(T)$ & Cabacés, Masroig, Palma d'Ebre, Ulldemolins & 4 & $\begin{array}{l}1994 \\
1995 \\
1996\end{array}$ & 12 & 12 & 4 \\
\hline & Garriges (L) & Borges Blanques, Cervià, Maials, Vinaixa & 4 & $\begin{array}{l}1994 \\
1995 \\
1996\end{array}$ & 63 & 21 & 63 \\
\hline & Montsià (T) & Alcanar & 1 & 1995 & 1 & 1 & 1 \\
\hline & Anoia (B) & Hostalets de Pierola & 1 & 1995 & 1 & 1 & 1 \\
\hline & Terra Alta (T) & Batea & 1 & 1995 & 1 & 1 & 1 \\
\hline Aragón & Zaragoza & Almunia Doña Godina & 1 & 1995 & 2 & 1 & 2 \\
\hline Andalucía & Jaén & Mengibar, Villacarrillo & 1 & 1995 & 3 & 3 & 3 \\
\hline & Córdoba & La Carlota & 1 & 1995 & 1 & 1 & 1 \\
\hline
\end{tabular}

$T=$ Tarragona; $L=$ Lleida; $B$ = Barcelona 
zonas catalanas, Córdoba, Jaén y Zaragoza (ver Tabla I). Todas las muestras de aceite se analizaron químicamente. Para la caracterización sensorial se utilizaron las recogidas en plena campaña (15 de diciembre). En el caso de los aceites de Garrigues, se cataron las 63 muestras recogidas, mientras que sólo 21 se analizaron químicamente. En el caso de Andalucía y Aragón, al disponer de menos muestras, se pretende ofrecer una caracterización preliminar de las mismas.

Los parámetros elegidos para caracterizar los aceites de esta variedad han sido los siguientes: acidez (\% de ácido oleico), absorción al U. V. $\left(\mathrm{K}_{270}\right)$, composición en ácidos grasos (\%), contenido en polifenoles totales (ppm de ácido cafeico, Vázquez Roncero et al., 1975), amargor $\left(\mathrm{K}_{255}\right.$, Gutiérrez Rosales et al., 1992), estabilidad del aceite (método Rancimat, en horas a $120^{\circ} \mathrm{C}$ ) y caracterización sensorial (método Panel Test, según normativa de la CEE núm. 2568/91).

Los resultados se analizan estadísticamente, calculando la media y desviación estándar de cada parámetro, para las distintas zonas estudiadas. Con los aceites obtenidos en Cataluña se realiza un análisis de la varianza, considerando los factores zona, año y su interacción. Posteriormente, tanto para los datos químicos como para los sensoriales, se realiza un
"Análisis de Componentes Principales», con el fin de comparar los aceites de las distintas zonas. Dicho análisis se realiza sobre los datos medios de cada zona y utilizando la matriz de correlaciones, aplicando el procedimiento PRINCOMP del programa estadístico SAS v. 6,08. El grado de similitud entre los aceites de las distintas zonas estudiadas se realiza mediante un Análisis de Clasificación Numérica aplicando el método del Centroide, donde las distancias entre clases se definen como separaciones entre individuos medios, mediante el procedimiento CLUSTER del programa estadístico anterior, considerando en cada caso el peso de las muestras de cada zona.

\section{RESULTADOS Y DISCUSIÓN}

Los resultados correspondientes a la caracterización de los aceites vírgenes de «Arbequina» producidos en Cataluña se exponen en la Tabla II, que incluye también una caracterización más parcial de los aceites de otras zonas de España. Se observa que cada zona de producción presenta unas características propias. Estos resultados son bastante parecidos a los encontrados en la revisión bibliográfica realizada con

Tabla II

Características medias ( \pm error estándar) de aceites vírgenes de «Arbequina» obtenidos en distintas zonas oleícolas de Cataluña, Andalucía y Aragón. Período 1994 a 1996

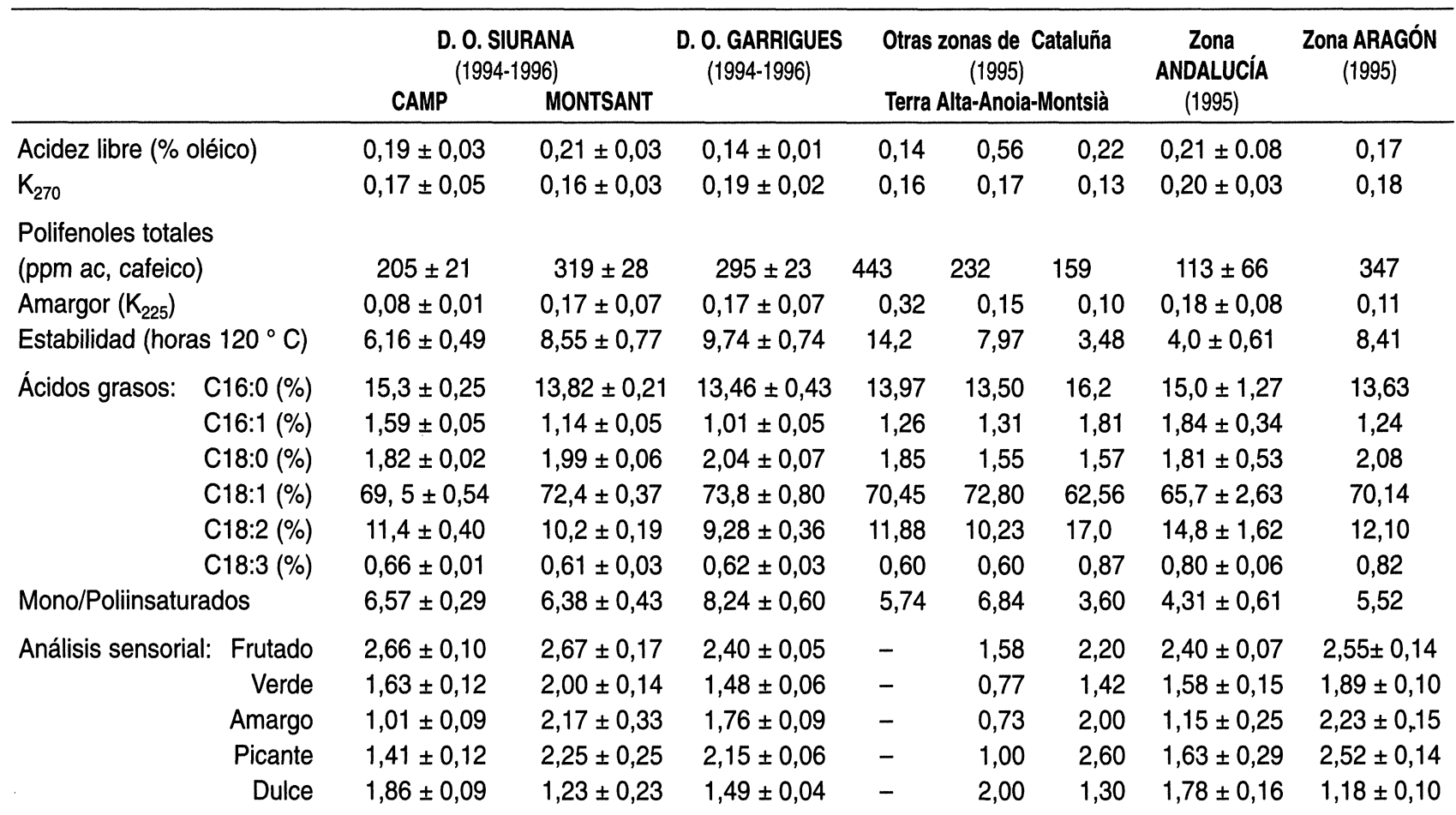


temas de calidad de sus aceites (Tablas III y IV). La Tabla $V$ muestra que, dentro de Cataluña, el efecto "zona» es altamente significativo en casi todos los parámetros analizados, a excepción del atributo sensorial verde. El efecto «año», sin embargo, sólo se ha mostrado significativo en el caso del contenido en ácido oleico, linoleico, la relación Mono/Poliinsaturados $(\mathrm{M} / \mathrm{P})$, el nivel de polifenoles, la estabilidad, el frutado de aceituna y la intensidad del atributo manzana. En ningún caso la interacción «zona $x$ año» se ha mostrado significativa, salvo para los atributos frutado y picante.

\subsection{Características físico-químicas}

Los aceites de oliva virgen analizados corresponden a la categoría comercial «Extra», presentando las siguientes características analíticas: una acidez inferior a $0,5^{\circ}$ y un $\mathrm{K}_{270}$ inferior a 0,25. La absorbancia al ultravioleta a $270 \mathrm{~nm}$ está relacionada, además de con el estado oxidativo, con el color de los aceites, dando valores elevados los verdes y bajos los amarillos claros (Uceda y Hermoso, 1994). Así los aceites provenientes de las zonas de Andalucía, Aragón y D. O. Garrigues tienen una mayor $\mathrm{K}_{270}$, y en consecuencia son de color más verdoso que los aceites de las otras zonas estudiadas.

La composición en ácidos grasos de los aceites estudiados varía de una zona de cultivo a otra (ver Tablas II, III y V). Los aceites de las D. O. Garrigues y Siurana-Montsant se caracterizan por tener un elevado contenido de oleico (72-74\%) y medio en ácidos linoleico (9-10\%) y palmítico (13-14\%), diferenciándose en la relación Mono/Poliinsaturados (M/P), que es menor en la zona Siurana-Montsant $(6,4)$ frente a la de Garrigues (8,2). En la D.O. Siurana-Camp los aceites presentan un menor contenido en oleico $(69,5 \%)$, medio en linoleico (11\%) y alto en palmítico (15\%),

Tabla III

Características químicas del aceite virgen de «Arbequina» obtenido en distintas zonas oleícolas

\begin{tabular}{|c|c|c|c|c|c|c|c|c|c|}
\hline \multirow[t]{2}{*}{ Zona de cultivo } & \multicolumn{4}{|c|}{ Ácidos grasos (\%) } & \multirow{2}{*}{$\begin{array}{c}\text { Relación } \\
\text { mono/poli- } \\
\text { insaturados }\end{array}$} & \multirow[t]{2}{*}{ K270 } & \multirow{2}{*}{$\begin{array}{c}\text { Polifenoles } \\
\text { (ppm ac. cafeico) } \\
{\text { secano }{ }^{a} \text { y regadío }}^{b}\end{array}$} & \multirow[t]{2}{*}{$\begin{array}{l}\text { Estabilidad horas } \\
98,8^{\circ} C^{\star} \text { y } 120^{\circ} C^{*}\end{array}$} & \multirow[t]{2}{*}{ Referencia } \\
\hline & C16:0 & C18:0 & C18:1 & C18:2 & & & & & \\
\hline \multicolumn{10}{|l|}{ Cataluña: } \\
\hline Lleida & 13,5 & 1,9 & 70,2 & 11,4 & 5,9 & - & - & - & Frías et al., 1991 \\
\hline Garrigues & 13,6 & 2,4 & 72,7 & 9,0 & 7,5 & - & - & - & Graell, 1992 \\
\hline Garrigues & 13,0 & 2,4 & 72,5 & 9,5 & 7,1 & 0,11 & - & $10^{* *}$ & Tous y Romero, 1994 b \\
\hline Garrigues & 13,1 & 2,2 & 74,4 & 8,3 & 8,3 & 0,11 & $262^{a}$ & $11,5^{\star \star}$ & Romero et al., 1995 \\
\hline Siurana-Montsant & 11,3 & 2,1 & 74,3 & 9,5 & 7,2 & 0,11 & - & $9,8^{\star *}$ & Tous y Romero, 1994 b \\
\hline Siurana-Montsant & 11,7 & 2,2 & 74,6 & 8,5 & 8,0 & 0,11 & $202^{a}$ & $9,8^{* *}$ & Romero et al., 1995 \\
\hline Siurana-Camp & 13,9 & 1,7 & 69,8 & 10,8 & 7,1 & 0,10 & - & $7,4^{* *}$ & Tous y Romero, 1994 b \\
\hline Siurana-Camp & 13,2 & 1,9 & 72,4 & 9,3 & 7,2 & 0,10 & $161^{a}$ & $7,7^{\star *}$ & Romero et al., 1995 \\
\hline Siurana-Camp & 13,4 & 1,9 & 70,8 & 10,2 & 6,4 & - & - & $7,3^{\star \star}$ & Papaseit y Cabré, 1988 \\
\hline \multicolumn{10}{|l|}{ Andalucía: } \\
\hline Córdoba (1) & 17,3 & 1,6 & 62,3 & 14,9 & 3,9 & 0,10 & $195^{b}$ & $46,1^{*}$ & Humanes y Civantos, 1993 \\
\hline Córdoba & 15,8 & 1,5 & 65,2 & 13,4 & 4,9 & 0,10 & $181^{b}$ & $38^{*}$ & Uceda y Hermoso, 1994 \\
\hline Córdoba & 17,1 & 1,3 & 63,2 & 14,7 & 4,2 & 0,10 & $179 b$ & $41^{*}$ & Uceda et al., 1989 \\
\hline Córdoba (2) & 16,7 & 1,4 & 64,2 & 14,2 & 4,2 & - & - & - & Civantos et al., 1992 \\
\hline La Carlota (Córdoba) (3) & 12,8 & 2,3 & 69,5 & 10,9 & 6,2 & 0,14 & - & $21^{*}$ & Delgado y Lobillo, 1995 \\
\hline La Carlota (Córdoba) & 14,3 & 1,6 & 65,0 & 12,8 & 4,9 & 0,15 & - & $21,9^{\star}$ & Delgado y Lobillo, 1995 \\
\hline Córdoba & - & - & - & - & - & - & $312^{a}, 156^{b}$ & $50,4^{a^{\star}} ; 31,5^{b^{*}}$ & Beltrán et al., 1995 \\
\hline \multicolumn{10}{|l|}{ Aragón: } \\
\hline Zaragoza (Almunia) & 10,9 & 2,0 & 76,3 & 7,9 & 9,1 & - & - & - & Gracia, 1995 \\
\hline Zaragoza (Almunia) & 13,1 & 2,2 & 70,8 & 10,9 & 6,1 & 0,12 & - & - & Gracia, 1996 \\
\hline \multicolumn{10}{|l|}{ Argentina: } \\
\hline Mendoza & 12,8 & 1,9 & 61,0 & 21,1 & 3,0 & - & - & - & Cattaneo, 1955 \\
\hline Córdoba & 16,3 & 0,7 & 61,9 & 16,5 & 4,0 & - & - & - & Cattaneo, 1955 \\
\hline
\end{tabular}

(1) Índice de Madurez $(\mathrm{IM})=1,84 ;$ (2) IM = 2,7, media de 6 muestras; (3) Media de 8 muestras 
con una relación M/P de 6,6, parecida a la de los aceites de la D. O. Siurana-Montsant. Los aceites de Andalucía presentaron, en 1995, una composición en ácidos grasos claramente distinta a los anteriores, así, el contenido en oleico es inferior al $66 \%$, el linoleico es superior al $14 \%$ y el palmítico oscila alrededor del $15 \%$, mientras que la relación $M / P$ es muy baja $(4,3)$, de acuerdo con la revisión bibliográfica realizada (Tabla III). Las muestras de Zaragoza (Aragón), Terra Alta (sur-oeste de Tarragona) y Anoia (Barcelona) presentaron en 1995 un cromatograma parecido a Siurana-Montsant, mientras que la del Montsià (sur de Tarragona) fue la que presentó el menor contenido en oleico $(62 \%)$ y el mayor de linoleico $(17 \%)$ y de palmítico (16\%). En general, se observa un inferior contenido en ácidos grasos saturados en las zonas más frías y de mayor altitud de Cataluña (Garrigues, SiuranaMontsant, Anoia y Terra Alta) y de Aragón, mientras que en las zonas más cálidas y septentrionales (Montsià y Andalucía) se obtienen aceites más ricos en linoleico y palmítico.
Tabla IV

Características sensoriales del aceite virgen de «Arbequina» obtenido en distintas zonas oleícolas

(Método panel-test, CEE 1991 n. 2568)

\begin{tabular}{lccc}
\hline & \multicolumn{3}{c}{ Zona de cultivo } \\
\cline { 2 - 4 } $\begin{array}{l}\text { Atributos } \\
\text { sensoriales }\end{array}$ & $\begin{array}{c}\text { D. 0. Siurana* } \\
\text { (Guerrero et al, 1996) }\end{array}$ & $\begin{array}{c}\text { D. O. Garrigues* } \\
\text { (Solé, 1995) }\end{array}$ & $\begin{array}{c}\text { Andalucía } \\
\text { (Uceda y Hermoso, 1994) }\end{array}$ \\
\hline Frutado & 2,43 & 2,16 & 3,12 \\
Verde hoja & 1,52 & 1,4 & 1,75 \\
Amargo & 1,06 & 1,8 & 0,62 \\
Picante & 1,60 & 1,7 & 0,62 \\
Dulce & 1,80 & 1,8 & 2,37 \\
Puntuación & 7,7 & 7,4 & 8,91 \\
\hline
\end{tabular}

* Media de 2 campañas; ${ }^{*}$ Media de 3 campañas

Tabla V

Efectos de la zona y del año en algunas características químicas y sensoriales de los aceites vírgenes de «Arbequina» obtenidos en distintas zonas de Cataluña. Período 1994-1996

Significación y porcentaje explicado de la varianza total

\begin{tabular}{|c|c|c|c|c|}
\hline \multirow[b]{2}{*}{ Características } & \\
\hline & Zona & Año & Zona x Año & Error \\
\hline \multicolumn{5}{|l|}{$\begin{array}{l}\text { Químicas: } \\
\text { • Acidos grasos }\end{array}$} \\
\hline $\mathrm{C} 16: 0$ & $25,6^{\star *}$ & 2,2 NS & $3,6 \mathrm{NS}$ & 68,7 \\
\hline C16:1 & $60,9^{\star \star}$ & $1,1 \mathrm{NS}$ & 3,2 NS & 34,9 \\
\hline C18:0 & $17,8^{* *}$ & $4,8 \mathrm{NS}$ & $0,5 \mathrm{NS}$ & 76,8 \\
\hline C18:1 & $34,4^{\star *}$ & $8,5^{\star}$ & 3,9 NS & 53,2 \\
\hline C18:2 & $27,7^{\star \star}$ & $41,4^{\star *}$ & 2,9 NS & 28,0 \\
\hline$M / P$ & $32,4^{* *}$ & $27,2^{\star \star}$ & $2,1 \mathrm{NS}$ & 38,3 \\
\hline - Polifenoles & 20,0 ** & $50,0^{* \star}$ & $3,3 \mathrm{NS}$ & 26,7 \\
\hline - Estabilidad & $26,3^{\star \star}$ & $25,5^{\star \star}$ & $0,6 \mathrm{NS}$ & 47,6 \\
\hline \multicolumn{5}{|l|}{ Sensoriales: } \\
\hline Frutado & $7,1^{* *}$ & $16,0^{* *}$ & $4,9^{*}$ & 72,0 \\
\hline Manzana & $42,8^{* *}$ & $5,0^{* *}$ & $1,6 \mathrm{NS}$ & 50,6 \\
\hline OFM & $15,1^{* *}$ & $3,6 \mathrm{NS}$ & $2,3 \mathrm{NS}$ & 79,0 \\
\hline Verde & $4,7 \mathrm{NS}$ & $3,7 \mathrm{NS}$ & $2,0 \mathrm{NS}$ & 89,5 \\
\hline Amargo & $18,6^{* *}$ & $2,6 \mathrm{NS}$ & $5,0 \mathrm{NS}$ & 73,7 \\
\hline Picante & $29,2^{\star *}$ & $3,8 \mathrm{NS}$ & $5,70^{*}$ & 61,3 \\
\hline Dulce & $20,2^{\star *}$ & $2,0 \mathrm{NS}$ & $5,1 \mathrm{NS}$ & 72,7 \\
\hline Otros & $7,7^{\star}$ & $1,7 \mathrm{NS}$ & $0,8 \mathrm{NS}$ & 89,8 \\
\hline
\end{tabular}

$\mathrm{M} / \mathrm{P}=$ relación Mono/Poliinsaturados $; \mathrm{OFM}=$ otras frutas maduras.

${ }^{\star \star},{ }^{\star}, N S=$ Significación al nivel de $p=0,01, p=0,05$ o no significativo, respectivamente. 
Estos resultados concuerdan con lo descrito por otros autores (Civantos et al., 1992), que han hecho constar que cuando más al sur se cultiva una variedad sus aceites aumentan en la proporción de ácidos grasos saturados, disminuye el oleico y aumenta el contenido en linoleico. La relación entre ácidos grasos insaturados y saturados es mayor en latitudes superiores y la relación oleico/linoleico aumenta igualmente. Este comportamiento podría responder a un alargamiento del ciclo de maduración, alcanzándose estadios de mayor grado de maduración en las latitudes más bajas.

Los polifenoles totales, antioxidantes naturales, también son muy variables según la zona, condiciones de cultivo y, principalmente, características del año (Tabla V), oscilando sus contenidos entre 200-350 ppm de ácido cafeico en las D. O. de Cataluña y Aragón, e inferiores en la zona de Andalucía (100-150 ppm), mostrando en ambos casos coeficientes de variación $(\mathrm{CV})$ muy elevados, del orden del $15-40 \%$. Sin embargo, en Andalucía existen otros trabajos donde obtienen mayores contenidos de polifenoles que los expuestos, del orden de $312 \mathrm{ppm}$ en secano y 156 ppm en regadío (Beltrán et al., 1995). Este parámetro está muy relacionado con las características sensoriales, al ser responsable del picor y amargor de los aceites de oliva virgen (Uceda y Hermoso, 1994).

La estabilidad oxidativa es un parámetro que nos mide la resistencia a la oxidación de un aceite, y tiene una gran importancia para valorar la calidad comercial y estado de conservación del mismo. Directamente relacionada con los polifenoles, las $\mathrm{D}$. O. de Cataluña y Aragón muestran una mayor estabilidad, entre 6-10 horas a $120^{\circ} \mathrm{C}$, con CV del $20-50 \%$. En Andalucía se obtienen estabilidades inferiores, del orden de 4-5 horas, aunque en la bibliografía consultada (ver Tabla III) se citan estabilidades superiores (equivalentes a unas 8-9 horas a $120^{\circ} \mathrm{C}$ ) a las obtenidas en este trabajo. Además del medio agrológico, sobre este parámetro inciden también varios factores, como son la presencia de antioxidantes naturales (polifenoles, tocoferoles, etc.) y la propia composición en ácidos grasos, principalmente, el ácido linoleico. En este sentido se han constatado elevadas correlaciones entre la estabilidad y la relación M/P (Tous y Romero, 1993; Jiménez y Uceda, 1995), y también con el contenido de polifenoles totales (Vázquez Roncero et al., 1975; Jiménez y Uceda, 1995). Los resultados de la Tabla II muestran una correlación entre estabilidad y polifenoles muy elevada $(r=0,86)$. La baja estabilidad de esta variedad, acentuada en alguna zona de cultivo, hace que la vida útil comercial de estos aceites sea muy inferior a la de otras variedades importantes («Picual»). Sin embargo, esta característica, claramente negativa, queda paliada por la poca cantidad de aceites de "Arbequina" que se comercializan actualmente, en relación a su demanda, de manera que el tiempo de almacenamiento (normalmente inferior a un año) nunca es tan largo como para que la baja estabilidad del aceite suponga una pérdida de calidad apreciable. Sin embargo, en una hipotética situación futura, con producciones muy superiores a las actuales, dicho factor debería ser tomado muy en cuenta.

El $K_{225}$ (amargor) nos muestra valores bastante bajos $(0,1-0,18)$, parecidos en las distintas zonas de cultivo estudiadas, y en concordancia con los bajos niveles de amargor observados en el análisis sensorial. En la comarca de la Terra Alta (Tarragona) se obtienen valores más altos $(0,32)$, superiores al límite 0,20 , a partir del cual se empieza a percibir el amargor con una mayor intensidad (Jiménez y Uceda, 1995).

Cuando se analizan conjuntamente estos datos físico-químicos, utilizando la técnica del "Análisis de Componentes Principales", se obtienen los resultados representados en la figura 1 . Con el fin de balancear los datos, previamente se han promediado las observaciones de cada zona. Para representar simultáneamente sobre un mismo plano factorial los puntosobservaciones y los puntos-variables, se han transformado las coordenadas de las observaciones en los ejes factoriales (PRIN K) por un factor $1 / \mathrm{p}^{1 / 2}$ con $p=8$ variables, para que sobre cada eje se tenga una dispersión respecto al origen comparable para las observaciones y para las variables (Judez, 1992). La primera componente principal (PRIN 1) permite explicar el $74 \%$ de la varianza total de los datos y está relacionada positivamente con el contenido en ácido oleico $(r=0,39)$, la relación $M / P(r=0,36)$ la estabilidad $(r=0,34)$ y el contenido en polifenoles $(r=0,32)$ y muestra una correlación negativa con el ácido palmitoleico $(r=-0,39)$, linoleico $(r=-0,38)$ y palmítico $(r=-$ 0,37 ). La segunda componente principal (PRIN 2) permite explicar un $13 \%$ de la varianza, estando correlacionada positivamente con el contenido en polifenoles totales $(r=0,58)$, la estabilidad $(r=0,41)$, y los ácidos linoleico $(r=0,35)$ y esteárico $(r=0,33)$ y negativamente con la relación $M / P(r=-0,43)$ y el ácido oleico $(r=-0,28)$.

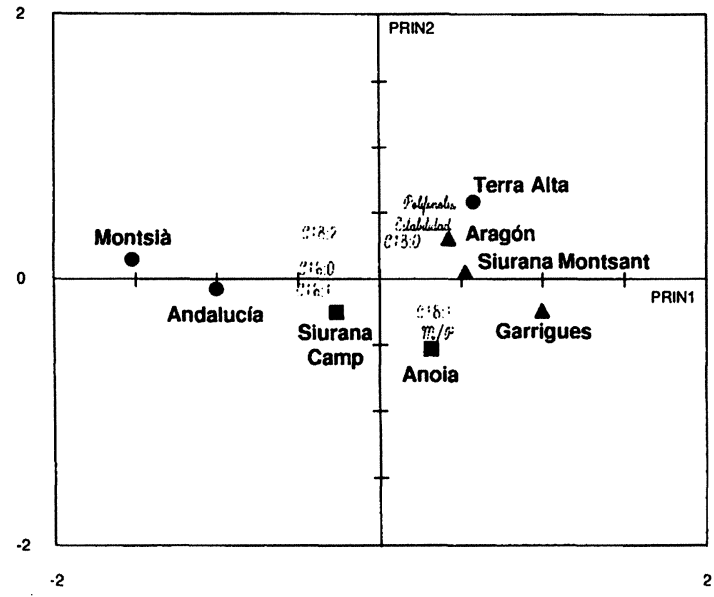

Figura 1

Características físico-químicas del aceite de oliva "Arbequina" procedente de distintas zonas de producción. Representación conjunta de las componentes principales (PRIN 1 y PRIN 2) de las observaciones (negrita) y de las variables (itálica). $\mathrm{M} / \mathrm{P}=-$ relación Mono/poliinsaturados 
Los resultados del análisis de agrupación de estos aceites se representan en el dendograma de la figura 2, siendo posible observar 4 tipos distintos de aceites: (a) un primer grupo (cuyas distancias normalizadas entre los individuos medios son siempre inferiores a $0,5)$ lo constituyen los aceites de Andalucía y Montsià, caracterizados por unos bajos contenidos en oleico, y altos en linoleico y palmítico; (b) un segundo grupo lo forman los de la D. O. Siurana-Camp y Anoia (distancia normalizada inferior a 0,30 ), que destacan por tener unos contenidos medios de oleico, linoleico y palmítico; (c) un tercer grupo (con distancias normalizadas inferiores a 0,50), formado por aceites de la D. O. Garrigues, D. O. Siurana-Montsant y Aragón, caracterizados por tener elevados valores de oleico, polifenoles y estabilidad; y (d) un cuarto grupo formado por los aceites de la Terra Alta, parecidos al grupo anterior pero con unos niveles de ácido esteárico claramente inferiores, que hacen que la distancia normalizada respecto al resto de aceites sea superior a 2. Aunque estos resultados pueden considerarse como bastante definitivos para el caso de los aceites producidos en Cataluña, sería preciso completarlos para los aceites de otras zonas españolas, donde el número de muestras y años estudiados ha sido inferior.

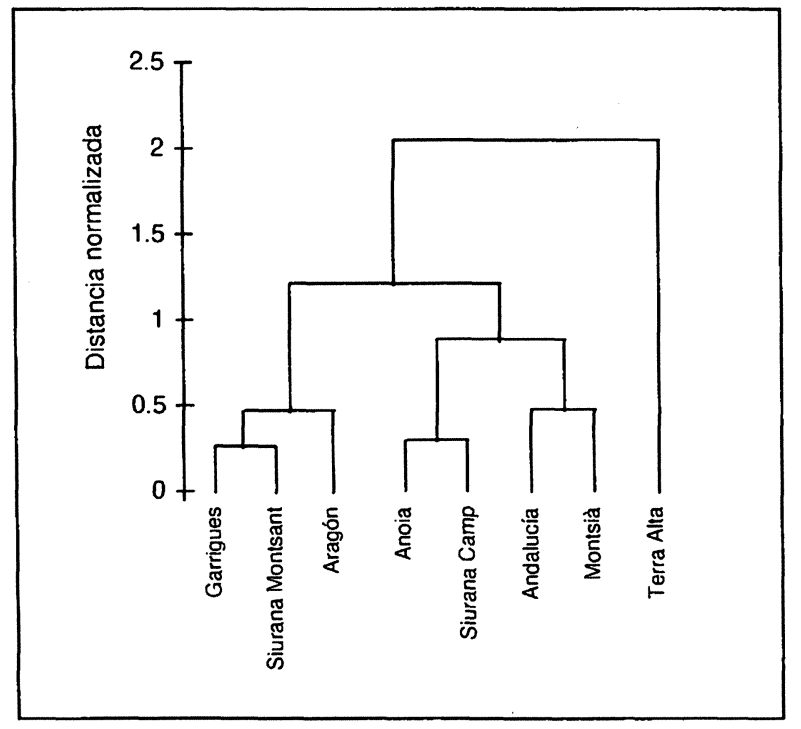

Figura 2

Características físico-químicas. Dendograma de distancias medias normalizadas entre aceites de oliva "Arbequina" procedentes de distintas zonas de producción

\subsection{Características sensoriales}

Las características sensoriales se reflejan en la Tabla II, en donde se presentan las intensidades medias de los aceites estudiados, durante las campañas de 1994 a 1996, respecto a sus atributos: frutado, verde, amargo, picante y dulce. En la Tabla $V$ se observa cómo los atributos manzana, otras frutas maduras, amargo, picante y dulce varían en función de la zona, mientras que el frutado depende más de las características del año. Se ha visto que los aceites frutados del tipo Garrigues, Siurana-Montsant y Aragón se diferenciaban con respecto a los tipos Siurana-Camp y Andalucía por ser más amargos y picantes, y menos dulces, de acuerdo con la revisión bibliográfica realizada (Tabla IV). En la cosecha de 1995 se observó un comportamiento distinto, posiblemente debido a las especiales características climáticas de este año (alta pluviometría), las cuales originaron una maduración muy precoz, principalmente en las zonas del interior de Cataluña, Garrigues y Siurana-Montsant (Tous et al., 1996). A la vista de lo expuesto, los perfiles sensoriales pueden variar según las características de la cosecha (clima, producción, etc.), principalmente en los atributos amargo, picante y dulce. También se conocen algunas correlaciones entre los diferentes atributos: el amargo y el picante tienen una relación inversa con el dulce y, directa, con el verde (Solé, 1995), constatados también en nuestro trabajo (Tabla VI).

Tabla VI

Principales correlaciones encontradas entre

algunos atributos sensoriales de muestras de aceite virgen de «Arbequina» $(N=88)$

\begin{tabular}{ccc}
\hline $\begin{array}{c}\text { Correlaciones } \\
\text { sensoriales }\end{array}$ & $\begin{array}{c}\text { Coef. de correlación } \\
(\mathbf{r})\end{array}$ & $\begin{array}{c}\text { Significación } \\
(\boldsymbol{\alpha})\end{array}$ \\
\hline Amargo- Picante & 0,72 & 0,0001 \\
Verde - Amargo & 0,57 & 0,0001 \\
Verde - Picante & 0,51 & 0,0001 \\
Amargo - Dulce & $-0,67$ & 0,0001 \\
Picante - Dulce & $-0,75$ & 0,0001 \\
Verde - Dulce & $-0,55$ & 0,0001 \\
Frutado - Verde & 0,80 & 0,0001
\end{tabular}

Los resultados del «Análisis de Componentes Principales" de las características sensoriales de los aceites estudiados, se presentan en las figuras 3 y 4 , habiéndose procedido como en el caso de las características físico-químicas. La primera componente principal (PRIN 1) permite explicar el $41 \%$ de la varianza total y está relacionada positivamente con el carácter dulce $(r=0,52)$ y negativamente con el amargo $(r=-0,53)$ y picante $(r=-0,52)$. La segunda componente principal (PRIN 2) explica un $33 \%$ de la varianza, estando correlacionada positivamente con la intensidad elevada del atributo verde $(r=0,60)$ y con el frutado de aceituna $(r=0,54)$. La tercera componente principal (PRIN 3) explica un $22 \%$ de la varianza y está correlacionada positivamente con los atributos manzana $(r=0,61)$ y otras frutas maduras $(r=0,50)$ y negativamente con otros atributos $(r=-0,51)$. 


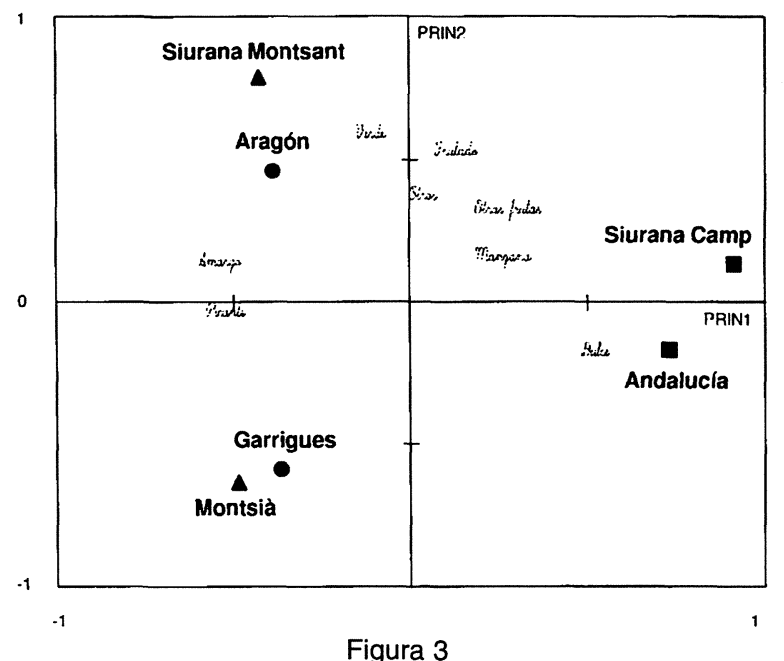

Características sensoriales del aceite de oliva "Arbequina" procedente de distintas zonas de producción. Representación conjunta de las componentes principales (PRIN 1 y PRIN 2) de las observaciones (negrita) y de las variables (itálica)

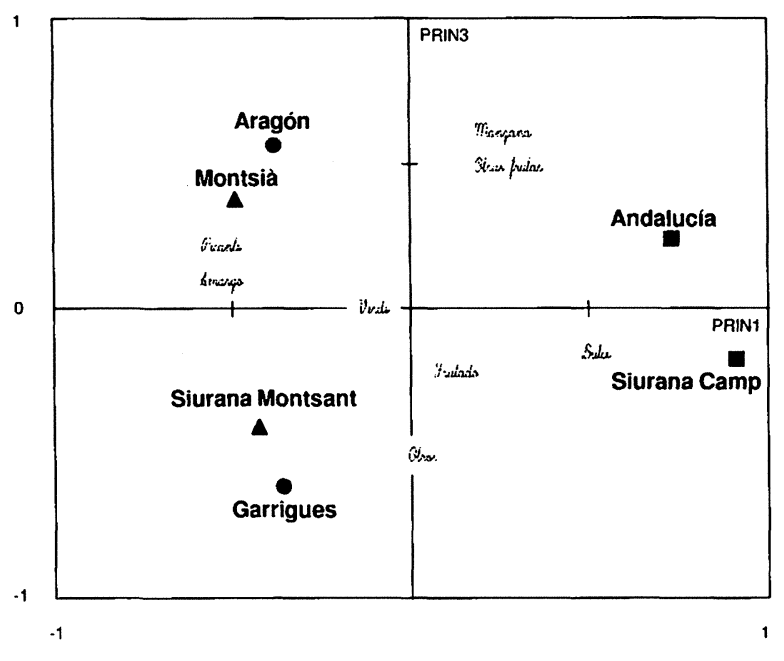

Figura 4

Características sensoriales del aceite de oliva "Arbequina». Representación conjunta de las componentes principales (PRIN 1 y PRIN 3) de las observaciones (negrita) y de las variables (itálica)

El análisis de agrupación se presenta, también, en la figura 5 con el dendograma obtenido. Se observa que los aceites de Andalucía son bastante parecidos a los de la D. O. Siurana-Camp (distancia normalizada inferior a 0,65), caracterizándose por ser más dulces y menos picantes y amargos que el resto de aceites de "Arbequina» analizados. Los otros aceites estudiados (Garrigues, Siurana-Montsant, Aragón y Monsià) son diferentes entre ellos (con distancias normalizadas mayores que 1,00). Los aceites de Garrigues son, en general, más amargos y picantes, mientras que los de Siurana-Montsant se caracterizan por ser los más frutados y verdes.

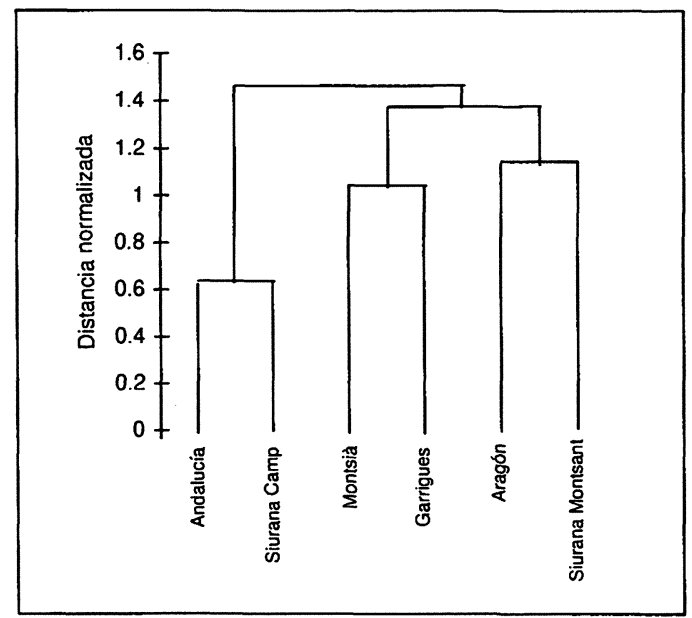

Figura 5

Características sensoriales. Dendograma de distancias medias normalizadas entre aceites de oliva "Arquina" procedentes de distintas zonas de producción

De todo lo expuesto anteriormente, se constata que la composición físico-química de estos aceites varía según la zona de producción y, también, puede oscilar según campañas, nivel de cosecha y grado de maduración de la aceituna; así, los aceites del tipo Garrigues, Siurana-Montsant y Aragón se diferencian, principalmente, respecto de los del tipo Siurana-Camp y Andalucía por tener un color amarillo más intenso, un mayor contenido en polifenoles y ácido oleico, así como un menor contenido en linoleico, todo lo cual conlleva una mayor estabilidad de los aceites producidos en estas zonas de interior, mayoritariamente en secano y con frecuentes déficits hídricos del noreste de España. Todos estos datos corroboran lo expuesto por diferentes autores (Civantos et al., 1992; Angerosa et al., 1995; Uceda y Hermoso, 1997), según los cuales los aceites obtenidos en zonas de montaña tienen unas características distintas que aquellos producidos en áreas de baja altitud, cercanos al litoral, y con elevadas temperaturas. Sensorialmente, los aceites de "Arbequina» son frutados, ligeramente amargos y picantes, y algo dulces, con algunas variaciones según zonas de cultivo, siendo organolépticamente similares los aceites de Andalucía y la D. O. Siurana-Camp.

El conocer las similitudes y diferencias entre los distintos tipos de aceites de «Arbequina» puede ser de gran interés para los envasadores, ya que pueden disponer de criterios objetivos para elaborar las mezclas comerciales destinadas a cubrir demandas diferenciadas.

\section{CONCLUSIONES}

Los principales aspectos a destacar de este trabajo, acerca de la caracterización físico-química y sen- 
sorial de los aceites de la variedad «Arbequina», son los siguientes:

- La variedad "Arbequina» produce aceites vírgenes de características químicas y sensoriales que varían significativamente en función de las condiciones medioambientales de la zona de producción.

- Los aceites obtenidos en las zonas "Garrigues" y «Siurana-Montsant» presentan características físico-químicas distintas que los de "Siurana-Camp", siendo los tres tipos de aceites distintos a nivel sensorial.

- La zona de Andalucía obtiene unos aceites vírgenes de "Arbequina» con características físico-químicas algo diferentes a las $D$. O. de Cataluña, que pueden ser debidas a la influencia del medio (menor latitud, temperaturas más elevadas, etc.) y del cultivo (alargamiento del ciclo de maduración, carga de los árboles jóvenes, riego, etc.) en la composición del aceite. Respecto de los caracteres sensoriales, su perfil se parece más a los aceites obtenidos en la zona «Siurana-Camp».

- La zona de Aragón produce aceites vírgenes de "Arbequina» de características físico-químicas y sensoriales parecidas a los aceites tipo «Garrigues» y «Siurana-Montsant».

\section{AGRADECIMIENTOS}

El presente trabajo ha sido financiado en parte por la sociedad "Olis de Catalunya, S. A.». Los autores quieren expresar su agradecimiento a la Estación de Olivicultura de Jaén (Mengíbar), a la cooperativa «EI Pilar» de Villacarrillo (Jaén) y a los técnicos $D$. Sebastián Delgado (La Carlota, Córdoba) y Dña. Soledad Gracia (D. G. de Aragón), por habernos facilitado muestras de aceite de «Arbequina», necesarias para la realización de este trabajo. Igualmente, agradecemos la colaboración de los miembros del panel de cata de Cataluña

\section{BIBLIOGRAFÍA}

Angerosa, F., Giancinto, L. D., Basti, C. y Serraiocco, A. (1996). - «Influenza della variabile "ambiente" sulla composizione degli oli vergini di oliva».- Riv. Italiana delle Sostanze Grasse 78, 461-467.

Aparicio, R., Albi, T., Cert, A. y Lanzón, A. (1988). -«Sistema experto SEXIA: Ecuaciones canónicas para caracterizar aceites de oliva españoles por variedades".- Grasas y Aceites 39, 219-228.

Aparicio R., Ferreiro, L. y Rodríguez, J. L. (1991). - "Caracterización de aceites de oliva vírgenes andaluces: Proyecto Sexia».- Colección: Informaciones Técnicas 13/91. Ed. D. G. de Investigación y Extensión Agrarias de la Junta de Andalucía. Sevilla.
Aparicio, R. (1995). - «El aceite de la variedad "Arbequina" en el contexto europeo". - I Simposi de l'Olivera Arbequina a Catalunya. Borges Blanques (Lleida), 131139.

Beltrán, G., Jiménez, A. y Uceda, M. (1995). -«Efecto del régimen hídrico de cultivo sobre la fracción fenólica del aceite de oliva de la variedad "Arbequina"».- I Simposi de l'Olivera Arbequina a Catalunya. Borges Blanques (Lleida), 153-155.

Cattaneo, P. (1955). - «Sobre la composición química de aceite de oliva argentino".- Actas Primera Conferencia Nacional de Olivicultura. Ed. Ministerio de Agricultura y Ganadería. Buenos Aires (Argentina), 391-396.

Cimato, A. (1990). - «La caratterizzazione del l'olio extravergine "tipico toscano" ".- Ed. Del Grifo. Montepulciano (Italia).

Cimato, A., Sani, G., Mattei, A. y Osti, M. (1990). - "Cultivars and environment as regulating factors in polyphenol and tocopherol contents of the Tuscan oilm.- Acta Horticulturae 286, 457-460.

Civantos, L., Contreras, R. y Grana, R. (1992). -“Obtención del aceite de oliva virgen».- Editorial Agrícola Española S. A., Madrid.

Cucurachi, A. (1965). - «Incidenza del fattore "varietà" delle olive sulla composizione acidica degli oli".- Rev. Ital. Sost. Grasse 42, 18-21.

Delgado, S. y Lobillo, C (1995). - «Características físico-químicas de aceite de oliva virgen "Arbequino" producido en Andalucia".- I Simposi de I'Olivera Arbequina a Catalunya. Borges Blanques (Lleida), 157-158.

Ferreiro, L. y Aparicio, R. (1992). - «Influencia de la altitud en la composición química de los aceites de oliva vírgenes de Andalucía. Ecuaciones matemáticas de clasificación”.- Grasas y Aceites 43, 149-156.

Frías, L., García Ortiz, A., Hermoso, M., Jiménez, A., Llavero, M. P., Morales, J., Ruano, M. T. y Uceda, M. (1991). - Analistas de laboratorio de almazara».Colección: Apuntes 6/91. Ed. D. G. de Investigación y Extensión Agrarias de la Junta de Andalucía. Sevilla.

García, J. M., Seller, S. y Pérez-Camino, M. C. (1996). - Influence of fruit ripening on olive oil quality».- J. Agric. Food Chem. 44, 3516-3520.

Gracia, S. (1995). -«Comunicación personal».

Gracia, S. (1996). -«El olivar y su aceite en Aragón».- Oleo (Especial SIO-96), 44-46.

Graell, J. (1992). - «Calidad del aceite de oliva virgen "D. O. Borjas Blancas". Influencia del proceso de extracción".II Salón Internacional Oleícola (SIO). Reus, 1-16.

Guerrero, L., Romero, A. y Tous, J. (1996). - «Análisis sensorial de aceites vírgenes de "Arbequina" en la D. O. Siurana (campañas 1994-1995)".- Datos no publicados.

Gutiérrez Rosales, F., Perdiguero, S., Gutiérrez, L. y Olías, J. M. (1992). - «Evaluation of the bitter taste in virgin olive oil».- J. M. Am. Oil. Chem. Soc. 69 (4), 394-395.

Humanes, J. y Civantos, M. (1993). -«Producción de aceite de oliva de calidad. Influencia del cultivo".- Colección: Apuntes 21/92. Ed. D. G. de Investigación, Tecnología y Formación Agroalimentaria y Pesquera de la Junta de Andalucía. Sevilla.

Inglese, P. (1994). - «Influencia de la variedad de las características cualitativas del aceite de oliva».- Olivae 54 (12), 42-47.

Jiménez, A. y Uceda, M. (1995). - «Características químicas y organolépticas del aceite de oliva virgen de la variedad "Arbequina" . - I Simposi de l'Olivera Arbequina a Catalunya. Borges Blanques (Lleida), 145-148.

Judez, L. (1982). - «Técnicas de análisis de datos multidimensionales».- Ed. MAPA, Secretaría General Técnica. Madrid.

Mousaa, Y. M. y Gerasopoulos, D. (1996). - «Effect of altitude on fruit and oil quality characteristics of "Mastoides" olives».- J. Sci. Food Agric. 71, 345-350. 
Papaseit, J. y Cabré, P. (1988). -«Relación entre las características de calidad y las medidas de estabilidad "Rancimat" en aceites de oliva".- Arxius de l'Esc. Sup. d'Agricultura de Barcelona 11, 33-42.

Parlati, M. V., Perri, E., Lombardo, N. y Palopoli, A. (1992). - "Caratteristiche qualitative degli oli di oliva vergini prodotti in Calabria».- "Olive Oil Quality» Congress. Firenze, 157-165.

Romero, A., Tous, J., Plana, J y Díaz, I. (1995). - «Caracterización físico-química de los aceites de oliva de la variedad "Arbequina"”. - I Simposi de I'Olivera Arbequina a Catalunya. Borges Blanques (Lleida), 157158.

Solé, M. A. (1995). - “Caracterización organoléptica de los aceites de oliva virgen extra de la variedad "Arbequina" en la zona de Les Garrigues". - I Simposi de I'Olivera Arbequina a Catalunya. Borges Blanques (Lleida), 149152.

Tous, J. y Romero, A. (1993). - «Variedades del olivo. Con especial referencia a Cataluña».- Ed. Fundación "La Caixa"- AEDOS. Barcelona.

Tous, J. y Romero, A. (1994 a). - "Cultivar and location effects on olive oil quality in Catalonia (Spain)».- Acta Horticulturae 356, 323-326.

Tous, J. y Romero, A. (1994 b).- «Aceites catalanes. Denominaciones de origen", en Olivicultura. Ed. Fundación "La Caixa»- AGROLATINO. Barcelona, 105112.

Tous, J., Romero, A., Plana, J., Guerrero, L. y Díaz, I. (1996). - "Características físico-químicas y organolépticas de los aceites de oliva virgen, variedad "Arbequina", obtenidos en Cataluña y Andalucía". - IV Salón Internacional Oleícola (SIO). Reus, 125-138.

Uceda, M. y Frías, L. (1975). -“Épocas de recolección. Evolución del contenido graso del fruto y de la composición y calidad del aceite".- II Seminario Oleícola Internacional. Córdoba.

Uceda, M., Hermoso, M. y Frías, L. (1989).- «Cultivar influence on oil quality-related parameters".- Olea 20, 145.

Uceda, M. y Hermoso, M. (1994). - "Aceites andaluces. Denominaciones de origen", en: Olivicultura. Ed. Fundación "La Caixa"-AGROLATINO. Barcelona, 113120.

Uceda, M. y Hermoso, M. (1997). - «La calidad del aceite de oliva".- p. 540-564. En: D. Barranco; R. Fernández Escobar y L. Rallo (eds.). El cultivo del olivo. Ed. MundiPrensa. Madrid.

Vázquez Roncero, A., Janer del Valle, C. y Janer del Valle, M. L. (1975). - «Polifenoles naturales y estabilidad del aceite de oliva".- Grasas y Aceites 26, 14-18.

Recibido: Junio 1997 Aceptado: Octubre 1997 